Article

\title{
Platelet Rich STROMA, the Combination of PRP and tSVF and Its Potential Effect on Osteoarthritis of the Knee
}

\author{
Hieronymus P. Stevens ${ }^{1, *,+} \mathbb{\oplus}$, Joeri van Boxtel ${ }^{2,+}{ }^{,}$Robbert van Dijck ${ }^{3}$ and Joris A. van Dongen ${ }^{2}$ \\ 1 PRSkliniek.nl, Rotterdam-Vlaardingen, Churchillsingel 472, 3127 XB Vlaardingen, The Netherlands \\ 2 Department of Plastic Surgery, University of Groningen and University Medical Center Groningen, \\ 9713 GZ Groningen, The Netherlands; j.van.boxtel@umcg.nl (J.v.B.); j.a.van.dongen@umcg.nl (J.A.v.D.) \\ 3 Bergman Clinics, 4817 BL Breda, The Netherlands; r.vandijck@bergmanclinics.nl \\ * Correspondence: Info@prskliniek.nl; Tel.: +31-624-810-440 \\ + Shared first author.
}

Received: 30 May 2020; Accepted: 6 July 2020; Published: 8 July 2020

\begin{abstract}
Background: osteoarthritis (OA) of the knee is a degenerative disease accompanied by pain, reduced mobility and subsequent decrease in quality of life. Many studies on OA of the knee have reported that using an intercellular acting-derivate like platelet-rich plasma (PRP) results in a limited effect or none at all. Authors hypothesized that adding tissue-Stromal Vascular Fraction (tSVF) to PRP (Platelet Rich Stroma (PRS)) would reduce pain and improve functionality in osteoarthritis of the knee. (2) Methods: a consecutive case series of fifteen patients (aged 43-75 years) suffering from OA of the knee (Kellgren-Lawrence stage two to three) were treated with a single injection of autologous PRS. tSVF was mechanically isolated by means of the fractionation of adipose tissue (FAT) procedure. Clinical evaluation was done using a visual analogue score (VAS) score, an adapted Western Ontario and McMaster Universities Osteoarthritis index (WOMAC) and Lysholm score at fixed time points: pre-injection as well as three, six and twelve months post injection. (3) Results: VAS and WOMAC scores improved significantly after twelve months $(p<0.01$ and $p<0.05)$. Lysholm instability scores were also improved at twelve months $(p>0.05)$ in comparison to pre-injection measurements. No complications were seen in any of the patients. One patient was excluded due to a total knee arthroplasty. (4) Conclusions: a single injection with PRS for OA of the knee seems to lead to an improvement of function and simultaneous reduction of pain and joint stiffness for a period of twelve months. Further controlled trials are required to determine the optimal treatment regimen and evaluate long-term results.
\end{abstract}

Keywords: SVF; PRP; knee osteoarthritis; regenerative

\section{Introduction}

Osteoarthritis (OA) of the knee is a chronic inflammatory disease that results in degeneration of cartilage in the joint, often accompanied by persistent pain, joint deformities and stiffness [1]. Combinations of these symptoms can lead to reduced mobility and a decreased quality of life [1,2]. The lifetime risk to develop symptomatic knee OA is $45 \%$ at the age of 85 years and is expected to increase because of increased obesity and aging in the Western World population [3]. In 2011, OA was the second most expensive condition treated in American hospitals, accompanied by a total cost of almost $\$ 15$ billion dollars per year [4]. In $50 \%$ of the cases, total knee arthroplasty was the reason for hospitalization [5]. The total cost related to the treatment $\mathrm{OA}$ is presumed to increase even more. This would be mostly due to the fact that the number of knee replacements is expected to increase by $673 \%$ in the USA by the year $2030[6,7]$. 
To date, most available treatments to decrease symptoms and the progressive character of OA are pharmacological and surgical treatments, e.g., anti-inflammatory or analgesic medication and joint replacement [8,9]. Ultimately, arthroplasty of the knee is the only available treatment resulting in a significantly improved mobility and reduction of pain, despite the risk for new postoperative sequelae (e.g., problems with kneeling, persistent pain and pain during walking) [10]. Arthroplasties are expensive and are associated with a long recovery period. Foote et al. found a return to work after total knee arthroplasty (TKA) of 12 weeks [11]. Multiple studies have been published focusing on new low-cost, regenerative and pain reducing therapies with a quick recovery time, e.g., autologous substrates, such as platelet rich plasma (PRP) or stromal vascular fraction (SVF) $[8,9,12]$.

PRP can be easily obtained from whole centrifuged blood and is defined as a portion of plasma fraction of autologous blood that preferably has a 2 to 2.5 increase of platelet concentrations above baseline [9]. Platelets contain numerous different growth factors, e.g., insulin-like growth factor (IGF), transforming growth factor $\beta 1$ (TGF- $\beta 1$ ) and vascular endothelial growth factor (VEGF) [9]. These growth factors increase proliferation, migration, differentiation and angiogenesis of adipose derived mesenchymal or stromal cells (ASCs) [13,14].

ASCs are multipotent stromal cells that reside in SVF of adipose tissue as precursor cells (supra-adventitial cells and pericytes) and possess regenerative capacities $[15,16]$. SVF consists of many other non-adipocyte cell types as well e.g., fibroblasts, lymphocytes, periadventitial cells and endothelial cells [12]. SVF can be isolated enzymatically or non-enzymatically from adipose tissue [17]. Enzymatic isolation of SVF results in a single cell suspension without cell-to-cell nor cell-to-extracellular matrix-communications (ECM), the so-called cellular SVF (cSVF) $[17,18]$. In contrast, non-enzymatic or mechanical isolation of SVF results in a tissue-like SVF (tSVF) mainly depleted of adipocytes, while other cell types as well as ECM remain mostly preserved [17,18]. Administration of mechanically isolated tSVF is less time-consuming as well as less expensive than enzymatic isolated cSVF [17]. The combination of the multiple growth factors and cells in tSVF stimulate angiogenesis, promote proliferation of parenchymal cells, suppress apoptosis and modulate immune responses [17]. Furthermore, the extracellular matrix acts as a scaffold ensuring slow release of growth factors and probably increases cell survival [19]. Thus, tSVF is anticipated to regenerate damaged tissue and/or modulate inflammation. These two processes stand at the very base of OA.

It was hypothesized that combining PRP and mechanically dissociated lipoaspirate tSVF (Platelet Rich Stroma (PRS)) would lead to a more effective treatment of OA of the knee. Hereby a consecutive series of fifteen randomly selected patients is presented being treated for OA of the knee with a single injection of PRS.

\section{Material \& Methods}

\subsection{Patient Population}

A consecutive series of fifteen patients were treated for OA of the knee between 15 May, 2018 and 9 October, 2018 by the first author (HPS), respecting the Declaration of Helsinki. An orthopaedic surgeon (RvD) was referring and scoring all patients, pre- and post-injection. Patients were graded two to three according to the Kellgren-Lawrence classification. Previous treatments consisted of physiotherapy, injections with hyaluronic acid gels and/or corticosteroids. As patients presented themselves to our clinics with persistent pain and reduced mobility, they did not respond well enough to previous treatments. Patients with hormonal or haematological diseases, cancer, sepsis, antiaggregating therapy and/or smoking in their medical history were excluded. Informed consent and written approval, confirming willingness to have a treatment done with PRS, were obtained from all participants. 


\subsection{Preparation of Platelet-Rich Plasma}

Autologous Conditioned Plasma (ACP)-PRP was obtained from fifteen $\mathrm{ml}$ of venous blood using a double syringe system (Arthrex ${ }^{\circledR}$, ACP System, Naples, FL, USA) according to the manufactures protocol. Briefly, whole venous blood-without the addition of anti-coagulants-was centrifuged in a swing-out rotor centrifuge (Hettich, Rotofix 32 benchtop, Kirchenlengern, Germany) at 1500 rpm delivering $340 \mathrm{~g}$ for five minutes at room temperature. The obtained $5 \mathrm{cc}$ of PRP was aspirated into the inner syringe of the ACP double syringe system. The ACP double syringe system is known to create a platelet count 2-3x above the baseline [20].

\subsection{Lipoharvesting and the Fractionation of Adipose Tissue (FAT) Procedure}

After local infiltration with a modified Klein solution (lidocaine 2\%, epinephrine 1:200.000 and bicarbonate $6.8 \%) 30 \mathrm{cc}$ of lipoaspirate was harvested peri-umbilically using two Arthrex ACP double syringes and disposable instruments of the Arthrex ACA-kit. After a first round of centrifugation of $4 \mathrm{~min}$. at $2500 \mathrm{rpm}(769 \mathrm{~g})$, the oily and aqueous fractions were discarded and the remaining condensed lipoaspirate was transferred into one $10 \mathrm{cc}$ luer-lock syringe using a 3-way cock. Subsequently, this syringe was connected to another empty $10 \mathrm{cc}$ luer-lock syringe using a 1-hole disposable fractionator in between $(1 \times 1.4 \mathrm{~mm}$ hole, luer-to-luer transfer, Tulip Medical Systems, San Diego, CA, USA). Next the Fractionation of Adipose Tissue (FAT) procedure was performed as previously described $[19,21]$. After pushing the condensed lipoaspirate 30 times forward and back through the Fractionator, the mechanically dissociated lipoaspirate was transferred back into an ACP-syringe and centrifugated for a second time for $2 \mathrm{~min}$. at $2500 \mathrm{rpm}(769 \mathrm{~g})$. This yields a final $90 \%$ of oily fraction that can be easily removed leaving a $10-\mathrm{vol} \%$ fraction $(1 \mathrm{~mL})$ of $\mathrm{tSVF}$ in the larger outer ACP double syringe (Figure 1, arrow B).

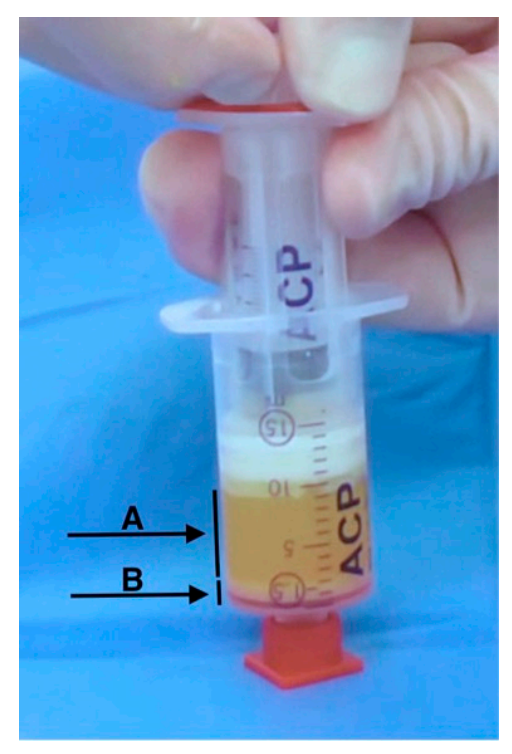

Figure 1. After the second round of centrifugation of the Autologous Conditioned Plasma (ACP)-syringe, filled with $10 \mathrm{cc}$ of fractionated lipoaspirate, the oily fraction of $90-\mathrm{vol} \%$ (arrow A) can be easily removed leaving a $10 \%$ fraction of $1 \mathrm{~mL}$ of tissue-Stromal Vascular Fraction (tSVF) (arrow B) in the larger outer ACP double syringe.

\subsection{Platelet Rich Stroma (PRS) Injection}

After performing the FAT-procedure, the inner syringe can be replaced with the syringe holding $5 \mathrm{cc}$ of PRP (Figure 2, arrow C). A total of $6 \mathrm{cc}$ of PRS is made in a closed setting, without the use of any chemicals or enzymes in a $45 \mathrm{~min}$. procedure. All patients received a single injection of PRS under local anaesthesia. The injection in the knee was performed under local anaesthetics. A total of $4 \mathrm{cc}$ of PRS 
was injected intra-articularly using a laterocranial retropatellar approach and the remaining $2 \mathrm{cc}$ were injected on either side of the patella tendon at the level of the tibia plateau (Figure 3). The laterocranial retropatellar (blinded) injection in the knee was proven to have good accuracy of approaching the intra-capsular joint [22].

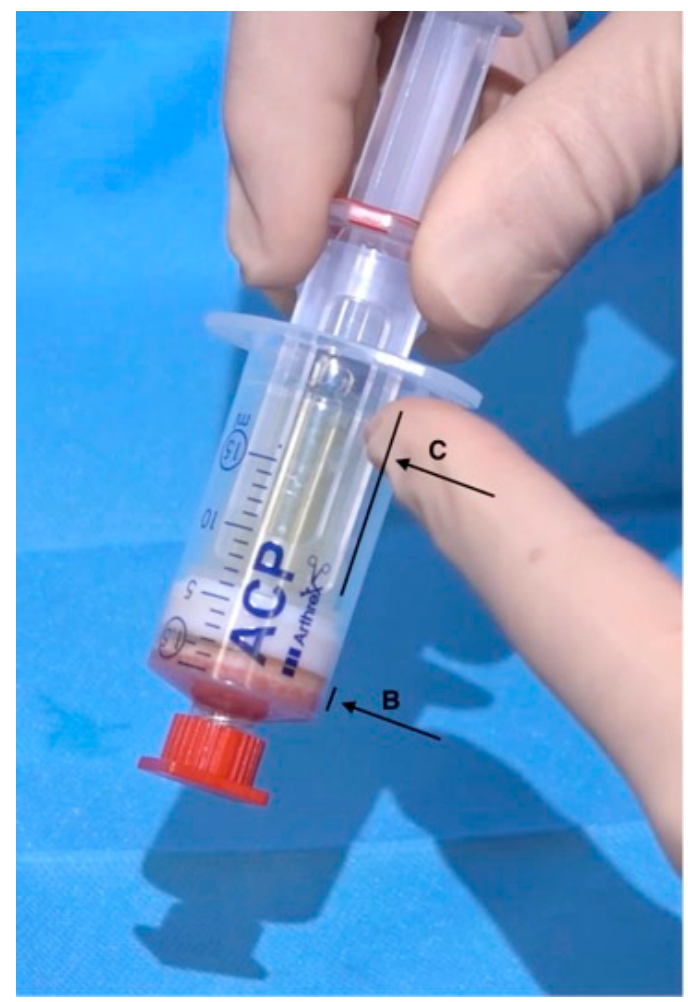

Figure 2. After performing the fractionation of adipose tissue (FAT)-procedure as described and obtaining 1 cc of tSVF (arrow B) as depicted in Figure 1, the inner syringe can be easily replaced with the same size syringe holding $5 \mathrm{cc}$ of platelet-rich plasma (PRP) (arrow C). A total of $6 \mathrm{cc}$ of PRS is made in a closed setting by gently emulsifying both fractions and finally bringing it in all in the inner syringe, ready for injection.

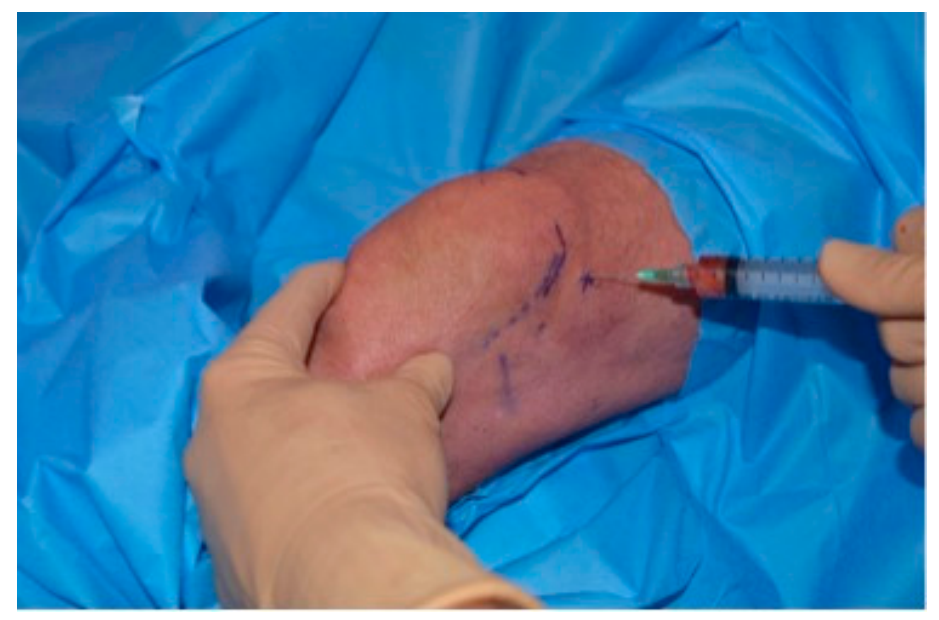

Figure 3. Laterocranial retropatellar approach for the injection of $4 \mathrm{cc}$ Platelet Rich Stroma (PRS) intra-articular into the left knee and on either side of the patella tendon $1 \mathrm{cc}$ of PRS was injected just above the tibia plateau. 


\subsection{Evaluation of the Effect of PRS on Knee Osteoarthritis}

Pre-injection, three months, six and twelve months post-injection changes were assessed using a visual analogue score (VAS), an adapted Western Ontario and McMaster Universities Osteoarthritis index (WOMAC) and the Lysholm instability score. The VAS-scores were also assessed at two and six weeks post-injection. WOMAC scores were related to pain (5 questions), stiffness (2 questions) and sociophysical functioning (17 questions). To prevent negative scores and allow for optimal graphical display, WOMAC scores were construed into the standard sum score. Additionally a higher score means a better outcome. The Lysholm instability score is a 100-point scoring system for examining a patient's knee-specific symptoms (including mechanical locking, instability, pain, swelling, stair climbing and squatting).

MRI images (T2) were assessed pre-injection and six months post-injection for cartilage integrity, bone marrow lesions, osteophyte formation, effusion, synovitis and meniscal abnormalities according to the Boston Leeds Osteoarthritis Knee Score (BLOKS) scoring system [23].

\subsection{Statistical Analysis}

The probability of a Gaussian distribution was tested with a D'Agostino-Pearson test for each outcome measure $(p>0.05)$. Next as there were four matched groups-comparing different follow up moments in time-assuming a normal distribution a repeated measure (RM) ANOVA test was performed $(p<0.05)$ followed by Tukey's multiple comparisons test $(p<0.05)$ using GraphPad Prism version 8.1.1 for OS X, GraphPad Software, San Diego, CA, USA, www.graphpad.com. All outcome measures for WOMAC function, stiffness, pain, VAS and Lysholm, were evaluated at three follow-up moments (pre-injection, three, six and twelve months postinjection). Data were expressed as mean $+/-$ standard deviation (SD).

\section{Results}

\subsection{Patient Demographics}

Fifteen patients ( 5 females, 10 males, ranging from $43-75$ years in age) were treated consecutively for OA of the knee. Twelve out of fifteen patients had been treated with corticosteroids before. Ten patients had prior treatments with hyaluronic acid injections. All patients had been using non-steroidal anti-inflammatory drugs regularly and all had received physiotherapy on a regular basis. The potential influence of confounding factors as corticosteroids and intensive physiotherapy was eliminated as none of the patients received any of such adjuvants after the injection with PRS during the course of the study. One patient was excluded from follow up due to a total knee arthroplasty after 8 months (TKA).

\subsection{Pain Significantly Decreased after Injection of PRS}

VAS scores presented passed the normality test $(p>0.05)$ in all cases except for pre-injection data. Differences in means observed were unlikely due to random sampling (RM ANOVA, $p<0.005$ ). Mean pre-injection VAS scores for pain significantly decreased from $4.83 \pm 1.89$ to $3.05 \pm 2.06$ twelve months post-injection (Tukey's multiple comparisons test, $p<0.01$ ). After six months, a small non-significant increase in mean VAS scores for pain was seen as compared to three months post-injection (Figure 4). 


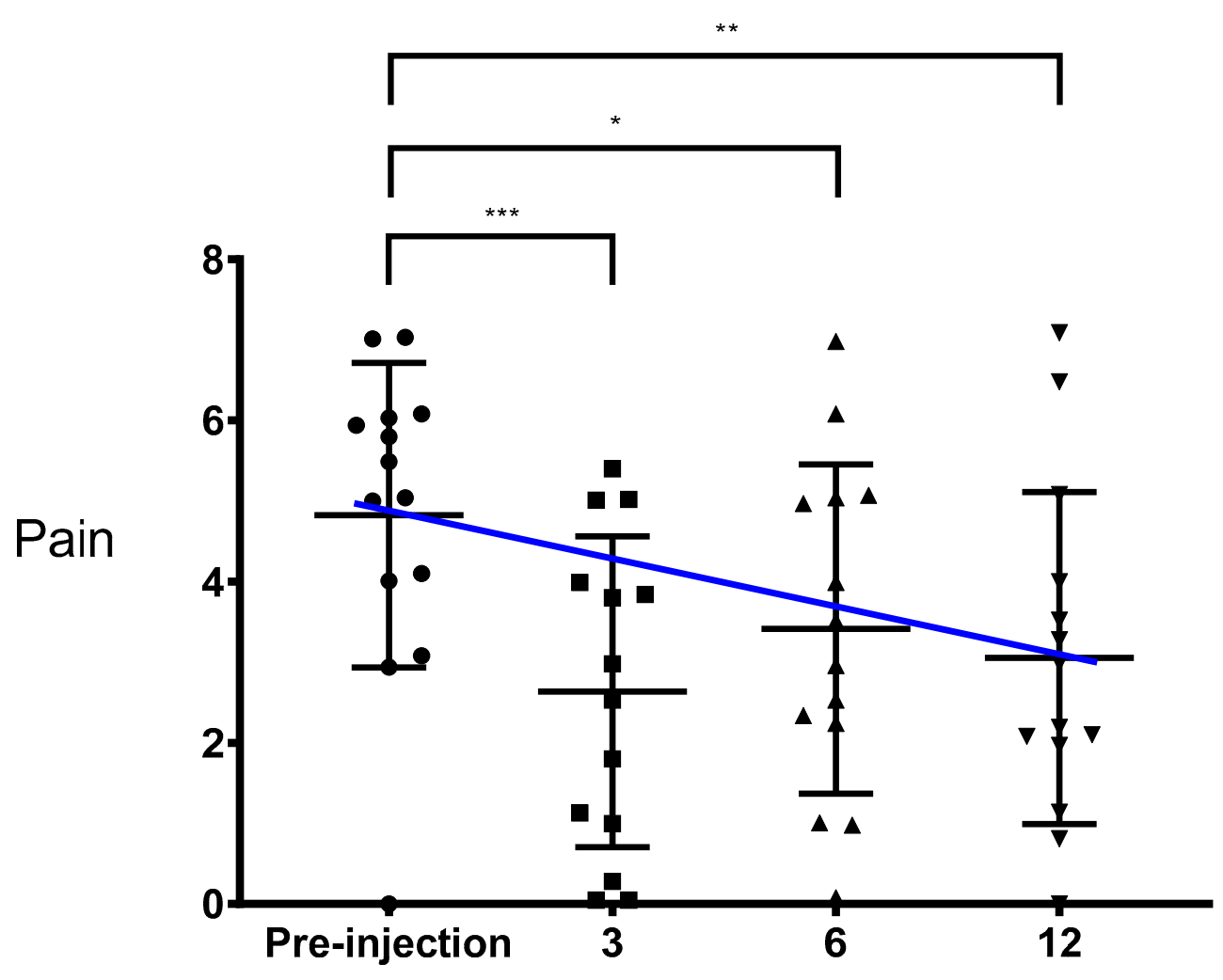

Time in Months

Figure 4. Visual analogue score (VAS) scores for pain of the osteoarthritic knee treated with PRS. A lower VAS score corresponds to less pain. Results are expressed as mean \pm standard deviation. Only significant results are marked. PRS = platelet rich stroma; ${ }^{*}=p<0.05 ;{ }^{* *}=p<0.01 ;{ }^{* * *}=p<0.001$.

WOMAC pain scores all passed the normality test $(p>0.05)$. Differences in means observed were unlikely due to random sampling (RM ANOVA, $p<0.0001$ ). Mean pre-injection WOMAC scores for pain significantly improved from $61.07 \pm 11.30$ to $74.64 \pm 15.38$ twelve months' post-injection (Tukey's multiple comparisons test, $p<0.05$, Figure 5). 


\section{WOMAC Pain}

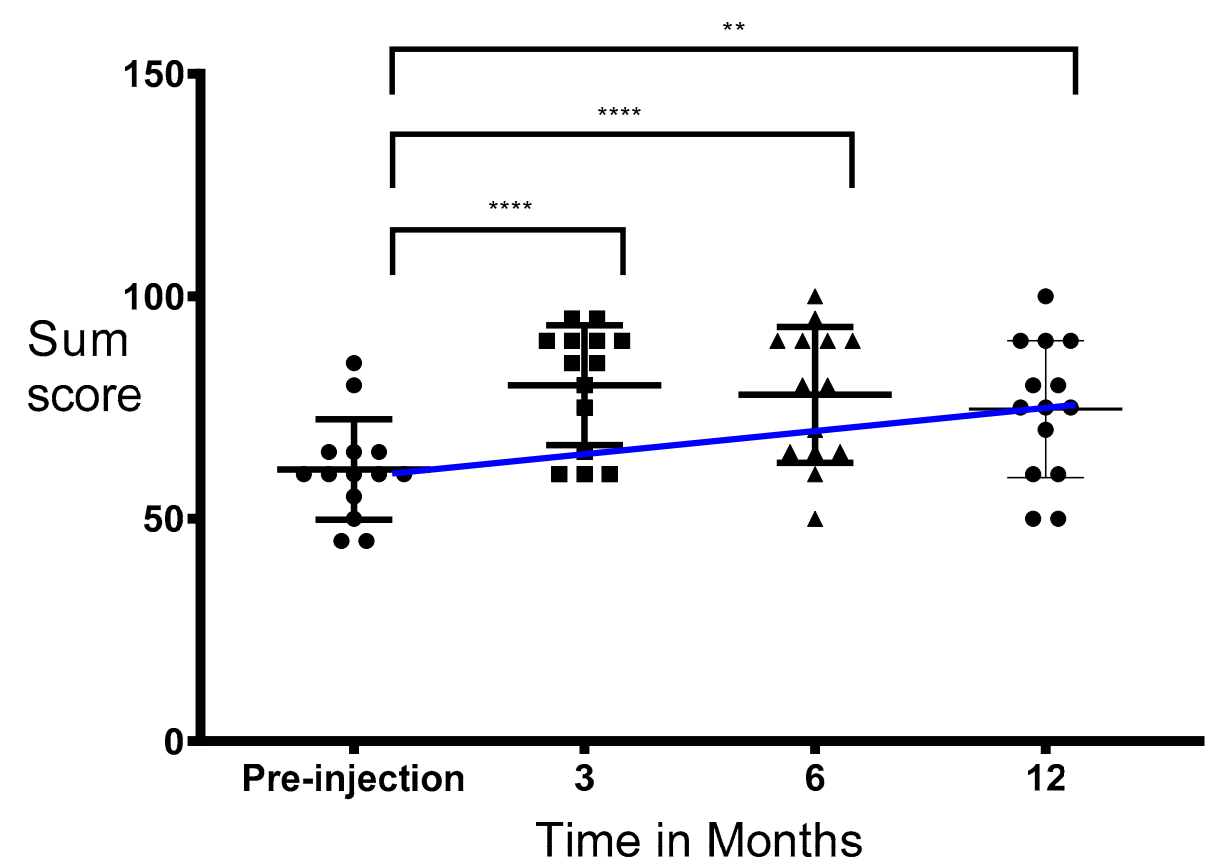

Figure 5. Western Ontario and McMaster Universities Osteoarthritis index (WOMAC) pain scores of the osteoarthritic knee treated with PRS. WOMAC scores were construed into a sum score. A higher WOMAC score means less pain. Results are expressed as mean \pm standard deviation. Only significant results are marked. PRS $=$ platelet rich stroma; ${ }^{* *}=p<0.01 ; * * * * p<0.0001$.

\subsection{Functionality and Stiffness of the Knee Significantly Improved after Injection of PRS}

The WOMAC data presented for function and stiffness all passed the normality test $(p>0.05)$. Differences in means observed were unlikely due to random sampling (RM ANOVA, $p<0.0001$ ). Mean WOMAC scores for function improved from $62.39 \pm 14.49$ to $79.31 \pm 15.17$ twelve months post-injection (Tukey's multiple comparisons test, $p<0.001$, Figure 6). A similar trend was seen in WOMAC scores for stiffness and Lysholm instability scores. WOMAC scores for stiffness significantly improved from $56.25 \pm 19.46$ pre-injection to $68.75 \pm 20.07$ twelve months post-injection (Tukey's multiple comparisons test, $p<0.05$, Figure 7). 


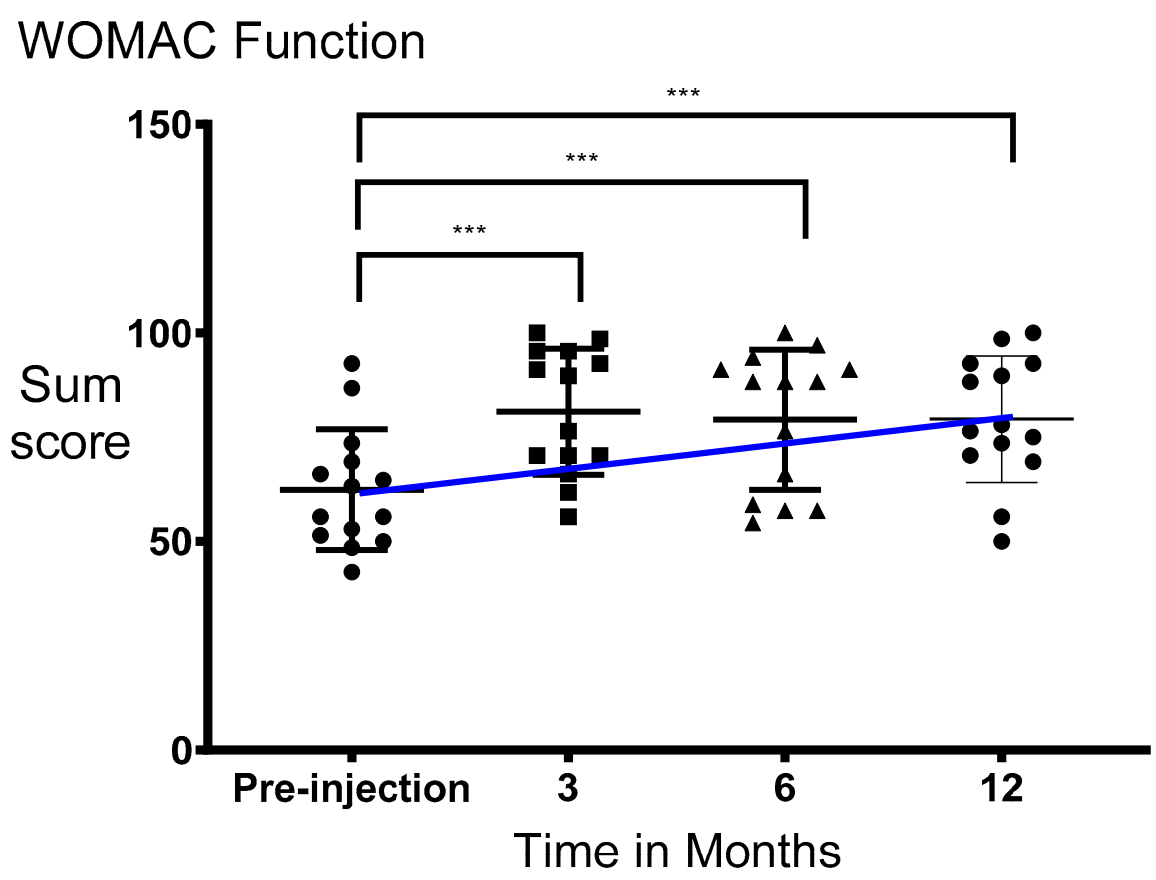

Figure 6. WOMAC function scores of the osteoarthritic knee treated with PRS. WOMAC scores were construed into a sum score. A higher WOMAC score means a better function. Results are expressed as mean \pm standard deviation. Only significant results are marked. PRS = platelet rich stroma; $* * *=p<0.001$.

\section{WOMAC Stiffness}

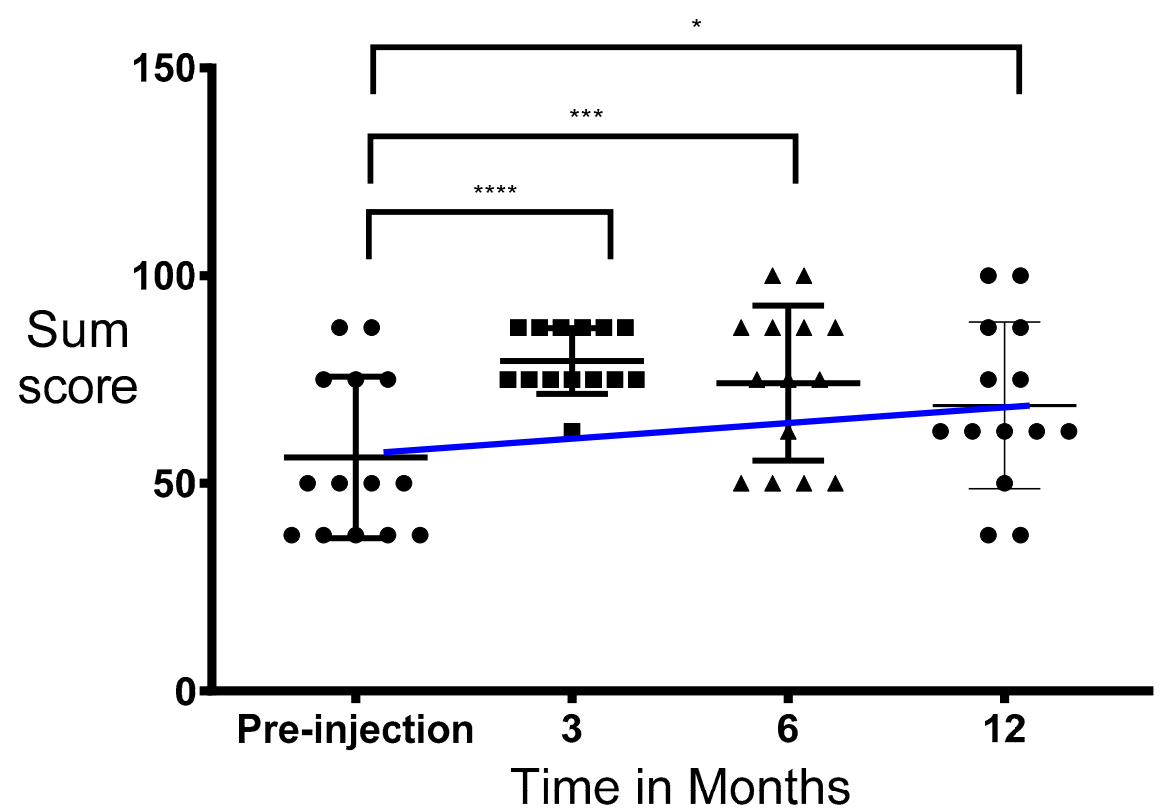

Figure 7. WOMAC stiffness scores of the osteoarthritic knee treated with PRS. WOMAC scores were construed into a sum score. A higher WOMAC score means less stiffness. Results are expressed as mean \pm standard deviation. Only significant results are marked. PRS $=$ platelet rich stroma; ${ }^{*}=p<0.05$; $* * *=p<0.001 ; * * * *=p<0.0001$. 
The data for the Lysholm instability scores all passed the normality test $(p>0.05)$. Differences in means observed were unlikely due to random sampling (RM ANOVA, $p<0.0001$ ). Mean Lysholm instability scores improved from $57.43 \pm 11.20$ to $66.64 \pm 15.58$ after twelve months in comparison to pre-injection measurements (Tukey's multiple comparisons test, $p>0.05$, Figure 8). No downtime was noted for any of the treated patients. No complications were seen in any of the patients in or around the injected knee. Some bruised feeling in the donor site due to lipoharvesting was present in all patients for 2 to 5 days. VAS and WOMAC pain and function scores showed a stagnation and/or decline from three to six months in the results, this, however, was not significant. WOMAC stiffness scores and Lysholm instability scores showed a decline from, respectively, three to twelve months and six to twelve months; this, however was not significant.

\section{Lysholm Instability Score}

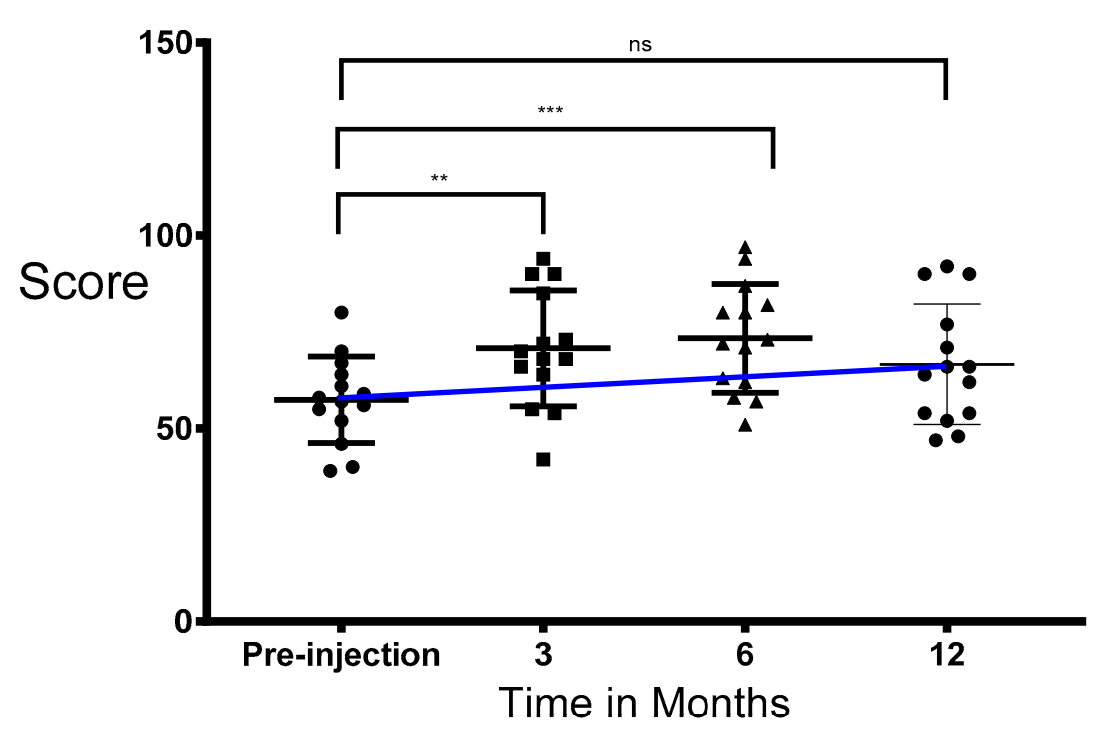

Figure 8. Lysholm Instability scores of the osteoarthritic knee treated with PRS. A higher Lysholm score means less instability. Results are expressed as mean \pm standard deviation. Only significant results are marked. PRS $=$ platelet rich stroma; $\mathrm{ns}=$ not significant; ${ }^{* *}=p<0.01{ }^{* * * *}=p<0.001$.

\subsection{No Significant Changes on MRI Were Seen after Injection of PRS}

Changes for previously existing cartilage abnormalities, bone marrow lesions, osteophyte formation, effusion, synovitis and meniscal abnormalities were not significant. However, changes in cartilage integrity grade 3 of the medial tibiofemoral compartment (rated from grade 0 to 3 ) were observed pre-injection in 8 out of 15 casus (53\%) and only still present in 6 out of 14 cases $(42 \%)$ at one-year post injection. Presence of synovitis decreased from 9 out of 15 cases $(60 \%)$ showing synovitis pre-injection to 6 out of $14(43 \%)$ after one year.

\section{Discussion}

A single injection of PRS in the knee suffering from OA appeared to significantly improve the physical and socio-emotional well-being of these patients up to twelve months post-injection. Shortly after injection, a steep decrease of pain and stiffness in the injected knee as well as improvement of physical function was seen in the majority of the patients. After three months post-injection, however, some recurrence of discomfort could be observed. This finding could be due to an increase in activities after six weeks, as the reduction of pain and previous limitations enabled recuperation of activities that up to then had to be minimalized. This suggests the timing and effect of (possible) plural injections should be analysed in the future. 
In a healthy joint, articular chondrocytes provide a balance between extracellular matrix (ECM) component synthesis and degradation by secretion of growth factors and cytokines [24]. In OA, the balance shifts towards ECM degradation through excessive production of pro-inflammatory cytokines and ECM degrading enzymes [25]. The imbalance in ECM remodelling results in cellular and oxidative stress, cartilage degradation and cell apoptosis [26]. Stressed cells produce more pro-inflammatory cytokines such as interleukin-1 $\beta$ (IL-1 $\beta$ ) and tumour necrosis factor-alpha (TNF-alpha) and endogenous molecules such as damage-associated molecular patterns (DAMPs) [27]. Binding of these cytokines and molecules to pattern-recognition receptors on cell types like chondrocytes activates the nuclear factor $k \beta(N F \kappa \beta)$ pathway [28]. The NFk $\beta$ pathway is involved in development of inflammation and pain and regulates tissue remodelling in joints by promoting secretion of matrix metalloproteinase (MMP-1, 3, 13) and a disintegrin and metalloproteinase with thrombospondin motifs 4 (ADAMTS4) [26]. Growth factors in PRP, e.g., hepatocyte growth factor, platelet-derived growth factor, IGF and TGF- $\beta 1$, inhibit the NFK $\beta$ signalling pathway and are therefore likely able to reduce the catabolic and anti-anabolic effects [26]. Downregulation of the NFK $\beta$ signalling results in a reduced production of ADAMTS4 and promotes production of type 2 collagen and proteoglycans in vitro $[29,30]$. The decrease in inflammation might result in decreased pain and, therefore, be responsible for restoring the balance in ECM remodelling.

Multiple preclinical and clinical studies have showed variable results from either PRP or SVF as a treatment for OA. Only recently, the combination of PRP and SVF is gaining interest [31-33]. The anti-inflammatory effects of PRP on osteoarthritic joints due to secretion of numerous growth factors can be strengthened by addition of non-manipulated ASCs being present in the form of tSVF. In tSVF, the ASCs are still attached to the vascular fibrous matrix [19]. ASCs are able to produce growth factors such as TGF- $\beta 1$ and IGF as well. It is, however, the combined injection of PRP and ASCs attached to their matrix that might be a prerequisite to allow for a synergistic effect. Growth factors secreted by platelets are capable of binding to the matrix, enabling slow release during the healing process [34]. Willemsen et al. presented a PRP induced dose-dependent increase of the proliferation rate of ASCs and a dose-dependent increase of several paracrine genes, such as TGF- $\beta 1$, IGF and fibroblasts growth factor 1 (FGF1) [35]. In contrast, gene expression of IL-1B showed a dose-dependent decrease [35]. Increased quantities of the anti-inflammatory growth factor TFG- $\beta 1$ and decreased amounts of the pro-inflammatory IL-1 $\beta$ might reduce joint inflammation. In addition, increased quantities of anti-apoptotic growth factors IGF and FGF1 might reduce secretion of inflammatory cytokines by chondrocytes. The role of the intact extracellular matrix (ECM) holding non-manipulated ASCs in their local niche might be more important than previously thought [34].

MRI data in this study showed no significant improvement of synovitis and the changes in the cartilage integrity. The inability to show cartilage or synovial improvement might be due to the small sample size or MRI imaging techniques used i.e., a Tesla 2.0. In regular hospitals, the Tesla 2.0 imaging technique has limited or no capacity to elucidate processes taking place on a cellular level. Possibly, a specific software protocol for imaging cartilage (which is expensive and therefore unavailable in most hospitals) could lead to more sensitive results. On the other hand, no formal proof exists of in vivo cartilage regeneration in humans as mechanism of reduced pain and improved joint function after treatment of SVF with or without PRP. These clinical improvements might be caused by a reduced anti-inflammatory state of the joint by decreasing the amount of prostaglandin E2. Subsequently, pain sensation might diminish and improve function of the affected joints without cartilage regeneration. It is well known, that many patients suffer from osteoarthritis e.g., pain of the affected joint without visible cartilage damage on imaging. The aforementioned mechanisms, to our opinion, support our preference of keeping this so called 'Trinity of Repair' (signal, repair cell i.e., ASCs and ECM) together.

Based on this consecutive case series alone, it is not realistic to state too firmly that improvements as presented can be ascribed to a synergistic effect of tSVF and PRP. The therapeutic effect of tSVF on knee OA might dominate the therapeutic effect of PRP or conversely [36]. Interestingly, the Lysholm instability score is the only (secondary) outcome measure with a non-significant improvement at twelve 
months. The association between instability and for example function i.e., more active patients-with simultaneously building muscle strength-has not been found to be significant [37]. Furthermore, reduced stiffness was associated with subjective instability in the knee [37]. As the WOMAC stiffness scores significantly improve at twelve months, it could be hypothesized, that due to less stiffness more subjective instability is experienced. This study is envisioned to provoke the interest to invest into, and setup, a larger clinical prospectively randomized controlled trial to address all these aspects of PRS as a treatment of OA against a control group with e.g., saline, for its potential effects, also to distinguish them from placebo effects that have been reported to be quite prominent sometimes [38].

Based on the results presented in this consecutive case series, the treatment of OA of the knee with PRS seems promising and deserves further investigation. To our opinion, it encourages to embrace the concept of 'repair before replace'. Offering a possible regenerative treatment of OA of the knee in an earlier stage — or even in the end stage aiming to postpone arthroplasty — might prove to be both very cost effective as well as resulting in increased quality of life.

Author Contributions: H.P.S. shared first author and corresponding author; designed and conceptualized study; role in the acquisition of data; revised the manuscript for intellectual content. J.v.B. shared first author; analysed the data; drafted the manuscript for intellectual content. R.v.D. second author; major role in the acquisition of data; revised the manuscript for intellectual content. J.A.v.D. senior author; designed and conceptualized study; partially drafted the manuscript for intellectual content; revised the manuscript for intellectual content. All authors have read and agreed to the published version of the manuscript.

Funding: This research was funded by Arthrex ${ }^{\circledR}$ in the form of ACA-kits and study support. This funding source had no role in the design of this study and did not have any role during its execution, analyses, interpretation of the data, or decision to submit results.

Conflicts of Interest: The authors report non-financial support and other from Arthrex Inc., Naples, Florida USA during the conduct of the study. H.P.S. and R.v.D. report personal fees from Arthrex Inc., Naples, Florida USA, as senior consultants outside the submitted work. H.P.S. also has a royalty agreement for the ACA-kit described. H.P.S. and R.v.D. are consultants for Arthrex Inc., Naples, FL USA. For this specific study, financial support was received (ACA-kits and study support).

\section{References}

1. Silverwood, V.; Blagojevic-Bucknall, M.; Jinks, C.; Jordan, J.L.; Protheroe, J.; Jordan, K.P. Current evidence on risk factors for knee osteoarthritis in older adults: A systematic review and meta-analysis. Osteoarthr. Cartil. 2015, 23, 507-515. [CrossRef] [PubMed]

2. Day-Williams, A.G.; Southam, L.; Panoutsopoulou, K.; Rayner, N.W.; Esko, T.; Estrada, K.; Helgadottir, H.T.; Hofman, A.; Ingvarsson, T.; Jonsson, H.; et al. A variant in MCF2L is associated with osteoarthritis. Am. J. Hum. Genet. 2011, 89, 446-450. [CrossRef] [PubMed]

3. Murphy, L.; Schwartz, T.A.; Helmick, C.G.; Renner, J.B.; Tudor, G.; Koch, G.; Dragomir, A.; Kalsbeek, W.; Luta, G.; Jordan, J.M. Lifetime risk of symptomatic knee osteoarthritis analysis and interpretation of data HHS public access. Arthritis Rheumatol. 2008, 59, 1207-1213. [CrossRef] [PubMed]

4. Torio, C.M.; Andrews, R.M. National Inpatient Hospital Costs: The Most Expensive Conditions by Payer, 2011: Statistical Brief \#160. In Healthcare Cost and Utilization Project (HCUP) Statistical Briefs; Agency for Healthcare Research and Quality (US): Rockville, MD, USA, 2006.

5. Neogi, T. The epidemiology and impact of pain in osteoarthritis. Osteoarthr. Cartil. 2013, 21, 1145-1153. [CrossRef]

6. Kurtz, S.; Ong, K.; Lau, E.; Mowat, F.; Halpern, M. Projections of primary and revision hip and knee arthroplasty in the United States from 2005 to 2030. J. Bone Joint Surg. Am. Vol. 2007, 89, 780-785. [CrossRef]

7. Kurtz, S.M.; Ong, K.L.; Lau, E.; Bozic, K.J. Impact of the economic downturn on total joint replacement demand in the United States: Updated projections to 2021. J. Bone Joint Surg. Am. Vol. 2014, 96, 624-630. [CrossRef]

8. Filardo, G.; Kon, E.; Longo, U.G.; Madry, H.; Marchettini, P.; Marmotti, A.; Assche, D.V.; Zanon, G.; Peretti, G.M. Non-surgical treatments for the management of early osteoarthritis. Knee Surg. Sport Traumatol. Arthrosc. 2016, 24, 1775-1785. [CrossRef]

9. Fibel, K.H. State-of-the-Art management of knee osteoarthritis. World J. Clin. Cases 2015, 3, 89. [CrossRef] 
10. Bala, A.; Penrose, C.T.; Seyler, T.M.; Mather, R.C.; Wellman, S.S.; Bolognesi, M.P. Outcomes after total knee arthroplasty for post-traumatic arthritis. Knee 2015, 22, 630-639. [CrossRef]

11. Foote, J.A.J.; Smith, H.K.; Jonas, S.C.; Greenwood, R.; Weale, A.E. Return to work following knee arthroplasty. Knee 2010, 17, 19-22. [CrossRef]

12. Gibbs, N.; Diamond, R.; Sekyere, E.O.; Thomas, W.D. Management of knee osteoarthritis by combined stromal vascular fraction cell therapy, platelet-rich plasma, and musculoskeletal exercises: A case series. J. Pain Res. 2015, 8, 799-806. [CrossRef] [PubMed]

13. Tobita, M.; Tajima, S.; Mizuno, H. Adipose tissue-derived mesenchymal stem cells and platelet-rich plasma: Stem cell transplantation methods that enhance stemness Mesenchymal Stem/Stromal Cells-An update. Stem Cell Res. Ther. 2015, 6, 1-7. [CrossRef] [PubMed]

14. Bourin, P.; Bunnell, B.A.; Casteilla, L.; Dominici, M.; Katz, A.J.; March, K.L.; Redl, H.; Rubin, J.P.; Yoshimura, K.; Gimble, J.M. Stromal cells from the adipose tissue-derived stromal vascular fraction and culture expanded adipose tissue-derived stromal/stem cells: A joint statement of the International Federation for Adipose Therapeutics and Science (IFATS) and the International Society for Cellular Therapy (ISCT). Cytotherapy 2013, 15, 641-648.

15. Corselli, M.; Chen, C.W.; Sun, B.; Yap, S.; Rubin, J.P.; Péault, B. The tunica adventitia of human arteries and veins as a source of mesenchymal stem cells. Stem Cells Dev. 2012, 21, 1299-1308. [CrossRef]

16. Lin, G.; Garcia, M.; Ning, H.; Banie, L.; Guo, Y.-L.; Lue, T.F.; Lin, C.-S. Defining stem and progenitor cells within adipose tissue. Stem Cells Dev. 2008, 17, 1053-1063. [CrossRef] [PubMed]

17. van Dongen, J.A.; Tuin, A.J.; Spiekman, M.; Jansma, J.; van der Lei, B.; Harmsen, M.C. Comparison of intraoperative procedures for isolation of clinical grade stromal vascular fraction for regenerative purposes: A systematic review. J. Tissue Eng. Regen Med. 2018, 12, e261-e274. [CrossRef] [PubMed]

18. van Dongen, J.A.; Stevens, H.P.; Harmsen, M.C.; van der Lei, B. Mechanical micronization of lipoaspirates: Squeeze and emulsification techniques. Plast. Reconstr. Surg. 2017, 139, 1369e-1370e. [CrossRef]

19. van Dongen, J.A.; Stevens, H.P.; Parvizi, M.; van der Lei, B.; Harmsen, M.C. The fractionation of adipose tissue procedure to obtain stromal vascular fractions for regenerative purposes. Wound Repair Regen. 2016, 24, 994-1003. [CrossRef]

20. Mazzocca, A.D.; McCarthy, M.B.; Chowaniec, D.M.; Dugdale, E.M.; Hansen, D.; Cote, M.P.; Bardley, J.P.; Romeo, A.A.; Arciero, R.A.; Beitzelet, K. The positive effects of different platelet-rich plasma methods on human muscle, bone, and tendon cells. Am. J. Sports Med. 2012, 40, 1742-1749. [CrossRef]

21. Van Dongen, J.A.; Gostelie, O.F.E.; Vonk, L.A.; De Bruijn, J.J.; Van Der Lei, B.; Harmsen, A.C.; Stevens, H.P. Fractionation of adipose tissue procedure with a disposable one-hole fractionator. Aesthet. Surg. J. 2019. [CrossRef]

22. Maricar, N.; Parkes, M.J.; Callaghan, M.J.; Felson, D.T.; O'Neill, T.W. Where and how to inject the knee-A systematic review. Semin. Arthritis Rheum. 2013, 43, 195-203. [CrossRef] [PubMed]

23. Hunter, D.J.; Lo, G.H.; Gale, D.; Grainger, A.J.; Guermazi, A.; Conaghan, P.G. The reliability of a new scoring system for knee osteoarthritis MRI and the validity of bone marrow lesion assessment: BLOKS (Boston-Leeds Osteoarthritis Knee Score). Ann. Rheumatol. Dis. 2008. [CrossRef] [PubMed]

24. Sophia Fox, A.J.; Bedi, A.; Rodeo, S.A. The basic science of articular cartilage: Structure, composition, and function. Sports Health 2009, 1, 461-468. [CrossRef] [PubMed]

25. Bonnans, C.; Chou, J.; Werb, Z. Remodelling the extracellular matrix in development and disease. Nat. Rev. Mol. Cell Biol. 2014, 15, 786-801. [CrossRef] [PubMed]

26. Andia, I.; Maffulli, N. Platelet-rich plasma for managing pain and inflammation in osteoarthritis. Nat. Rev. Rheumatol. 2013, 9, 721-730. [CrossRef]

27. Chen, G.Y.; Nuñez, G. Sterile inflammation: Sensing and reacting to damage. Nat. Rev. Immunol. 2010, 10, 826-837. [CrossRef]

28. Marcu, K.B.; Otero, M.; Olivotto, E.; Maria Borzi, R.; Goldring, M.B. NF-kappaB Signaling: Multiple angles to target OA. Curr. Drug Targets 2010, 11, 599-613. [CrossRef]

29. Van Buul, G.M.; Koevoet, W.L.M.; Kops, N.; Bos, P.K.; Verhaar, J.A.; Weinans, H.; Bernsen, M.R.; van Osch, G.J. Platelet-rich plasma releasate inhibits inflammatory processes in osteoarthritic chondrocytes. Am. J. Sports Med. 2011, 39, 2362-2370. [CrossRef] 
30. Wu, C.C.; Chen, W.H.; Zao, B.; Lai, P.-L.; Lin, T.-C.; Lo, H.-Y.; Shieh, Y.-H.; Wu, C.-H.; Deng, W.-P. Regenerative potentials of platelet-rich plasma enhanced by collagen in retrieving pro-inflammatory cytokine-inhibited chondrogenesis. Biomaterials 2011, 32, 5847-5854. [CrossRef]

31. Mehranfar, S.; Abdi Rad, I.; Mostafav, E.; Akbarzadeh, A. The use of stromal vascular fraction (SVF), platelet-rich plasma (PRP) and stem cells in the treatment of osteoarthritis: An overview of clinical trials. Artif. Cells Nanomed. Biotechnol. 2019, 47, 882-890. [CrossRef]

32. Bansal, H.; Comella, K.; Leon, J.; Verma, P.; Agrawal, D.; Koka, P.; Ichim, T. Intra-Articular injection in the knee of adipose derived stromal cells (stromal vascular fraction) and platelet rich plasma for osteoarthritis. J. Transl. Med. 2017, 15, 141. [CrossRef] [PubMed]

33. Hong, Z.; Chen, J.; Zhang, S.; Zhao, C.; Bi, M.; Chen, X.; Bi, Q. Intra-articular injection of autologous adipose-derived stromal vascular fractions for knee osteoarthritis: A double-blind randomized self-controlled trial. Int. Orthop. 2019, 43, 1123-1134. [CrossRef] [PubMed]

34. van Dongen, J.A.; Getova, V.; Brouwer, L.A.; Liguori, G.R.; Sharma, P.K.; Stevens, H.P.; van der Lei, B.; Harmsen, M.C. Adipose tissue-derived extracellular matrix hydrogels as a release platform for secreted paracrine factors. J. Tissue Eng. Regen. Med. 2019, 13, 973-985. [CrossRef] [PubMed]

35. Willemsen, J.C.N.; Spiekman, M.; Stevens, H.P.J.; Van Der Lei, B.; Harmsen, M.C. Platelet-Rich plasma influences expansion and paracrine function of adipose-derived stromal cells in a dose-dependent fashion. Plast Reconstr. Surg. 2016, 137, 554e-565e. [CrossRef] [PubMed]

36. Stevens, H.P.; Donners, S.; De Bruijn, J. Introducing platelet-rich stroma: Platelet-Rich Plasma (PRP) and Stromal Vascular Fraction (SVF) combined for the treatment of androgenetic alopecia. Aesthet. Surg. J. 2018, 38, 811-822. [CrossRef]

37. Wallace, D.T.; Riches, P.E.; Picard, F. The assessment of instability in the osteoarthritic knee. EFORT Open Rev. 2019, 4, 70-76. [CrossRef]

38. Saltzman, B.M.; Leroux, T.; Meyer, M.A.; Basques, B.A.; Chahal, J.; Bach, B.R., Jr.; Yanke, A.B.; Cole, B.J. The therapeutic effect of intra-articular normal saline injections for knee osteoarthritis: A meta-analysis of evidence level 1 studies. Am. J. Sports Med. 2017. [CrossRef]

(C) 2020 by the authors. Licensee MDPI, Basel, Switzerland. This article is an open access article distributed under the terms and conditions of the Creative Commons Attribution (CC BY) license (http://creativecommons.org/licenses/by/4.0/). 